\title{
A normal alveolar dead space fraction plus D-dimer assay ruled out suspected pulmonary embolism in the emergency department
}

\author{
Kline JA, Israel EG, Michelson EA, et al. Diagnostic accuracy of a bedside D-dimer assay and alveolar dead-space \\ measurement for rapid exclusion of pulmonary embolism: a multicenter study. JAMA 2001 Feb 14;285:761-8. \\ QUESTION: In patients with suspected pulmonary embolism (PE) who are in the \\ emergency department, can a normal alveolar dead space fraction (ADF) assessment \\ and a negative whole blood agglutination D-dimer assay exclude PE?
}

\section{Design}

Blinded comparison of test results for $\mathrm{ADF}$ and $\mathrm{D}$-dimer assay with radionuclide lung scanning $(\mathrm{V} / \mathrm{Q}$ scan), contrast enhanced computer tomography, and 6 month follow up plus selective use of venous ultrasonography and pulmonary angiography.

\section{Setting}

6 urban teaching hospitals in the US.

\section{Patients}

380 patients (mean age $50 \mathrm{y}, 70 \%$ women) with suspected PE. Inclusion criteria were age $>18$ years and not being transferred from another facility. Exclusion criteria were signs of circulatory shock, inability to breathe room air and maintain pulse oximetry reading of $<90 \%$, or failure to cooperate with volumetric capnometry and blood collection.

Description of tests and diagnostic standard ADF was calculated by using a computer algorithm with data from volumetric capnograms recorded for 2 minutes and arterial blood gas results. A test result of $<20 \%$ was considered to be normal. D-dimer testing was done at the bedside by using a whole blood agglutination assay (SimpliRED). The diagnostic standard included a $\mathrm{V} / \mathrm{Q}$ scan or a computed tomogram scan of the chest plus a 6 month follow up. For patients with a non-diagnostic $\mathrm{V} / \mathrm{Q}$ scan, venous ultrasonography and pulmonary angiography could also be done.

\section{Main outcome measures}

Sensitivity and specificity for both ADF and D-dimer assay results.

\section{Main results}

64 patients (18\%) had PE; these patients were older (56 $v 49 \mathrm{y})$ and less likely to report dizziness ( $16 \%$ v 31\%) than were patients without PE. In combination, the ADF and D-dimer test results had high sensitivity but poor specificity (table). Consequently, negative results from both tests effectively ruled out the diagnosis of $\mathrm{PE}$, even though positive results from both tests did not rule in the diagnosis.

\section{Conclusion}

A negative D-dimer test result plus a normal alveolar dead space fraction ruled out pulmonary embolism in symptomatic patients presenting to the emergency department.

\section{COMMENTARY}

No single non-invasive test has both high sensitivity and high specificity for PE. Consequently, in addition to an assessment of clinical probability (ie, low, moderate, or high), most patients with suspected PE require either several noninvasive tests or pulmonary angiography to rule in or rule out PE. Kline et al evaluate the accuracy of a whole blood D-dimer assay and measurement of ADF for the diagnosis of $\mathrm{PE}$. They confirm that this D-dimer assay has a moderate to high sensitivity for PE, the result is usually normal in the absence of PE, and a normal result is very helpful in ruling out PE. ${ }^{1}$ At best, ADF results were moderately predictive of PE. The authors indicate that the addition of ADF measurement to D-dimer testing resulted in a clinically important increase in sensitivity and a decrease in the negative likelihood ratio.

However, this conclusion may not be justified. Firstly, the trade off for detecting 3 cases of $\mathrm{PE}$ among the 216 patients with a normal D-dimer test result was that 49 false positive ADF results were obtained. Secondly, even these 3 patients may not have had acute PE; most emboli were diagnosed on the basis of high probability V/Q scans, which are associated with an appreciable proportion of false positive results. $\mathrm{ADF}$ is increased in patients with a high probability scan (ie, ventilated but not perfused lung segments) regardless of whether acute embolism exists. Further studies are required to determine whether and how measurement of ADF should be used as a diagnostic test for PE.

Clive Kearon, $\mathrm{MB}, \mathrm{PhD}$ Henderson Hospital, McMaster Clinic Hamilton, Ontario, Canada

1 Ginsberg JS, Wells PS, Kearon C, et al. Sensitivity and specificity of a rapid whole-blood assay for D-dimer in the diagnosis of pulmonary embolism. Ann Intern Med 1998;129:100611.

2 The PIOPED Investigators. Value of the ventilation perfusion scan in acute pulmonary embolism. JAMA 1990;263:2753-9. 